\title{
ESTUDO SOBRE A PERCEPÇÃO E A PRODUÇÃO \\ DO CONTRASTE DE VOZEAMENTO DA FALA \\ DE CRIANÇAS DE 3 ANOS
}

\section{A study on perception and production of voicing contrasts in the speech of 3-year old children}

\author{
Maria Teresa Rosangela Lofredo-Bonatto ${ }^{(1)}$, Sandra Madureira ${ }^{(2)}$
}

\begin{abstract}
RESUMO
Objetivo: apresentar um estudo da percepção auditiva que o adulto tem sobre as características das plosivas vozeadas e não-vozeadas em posição acentual tônica e pós-tônica produzidas por crianças de três anos. Métodos: inspeção fonética-acústica das características encontradas na produção das plosivas, quanto ao intervalo de obstrução, do burst, e análise quantitativa das medidas do Voice Onset Time das seis plosivas do Português Brasileiro (PB), em frases-veículo "Diga ___ baixinho" por quatro crianças da faixa etária dos três anos, três meninos e uma menina, falantes nativos do PB, com análise em espectrograma de banda larga no programa Praat. Posteriormente, submissão a 120 sujeitos adultos do corpus produzido pelas crianças, por meio de um teste de percepção, do tipo identificação. Resultados: encontradas na produção, características incomuns à fala do adulto, tanto para as vozeadas como as não-vozeadas. O teste revelou que os juízes perceberam as plosivas não-vozeadas como vozeadas pela presença de breathy vowel. As plosivas vozeadas sempre foram identificadas como vozeadas. O ponto articulatório, das plosivas bilabiais, alveolares e velares não interferiu no julgamento das plosivas. O teste ANOVA $(\mathrm{sig}=0,05)$ revelou que as plosivas foram identificadas corretamente quando apresentavam a presença de breathy vowel ou de barra de sonoridade com mais de $60 \%$ de vozeamento. A presença de aspiração não interferiu no julgamento das plosivas não-vozeadas. Conclusão: resultados indicaram que ocorreram dificuldades na sincronização entre o gesto glotal e o articulatório, que não interferiram na percepção desses sons, uma vez que as plosivas não-vozeadas que apresentaram aspiração foram corretamente avaliadas como não-vozeadas.
\end{abstract}

DESCRITORES: Criança; Medida de Produção da Fala; Espectrografia do Som; Percepção da Fala

\section{INTRODUÇÃO}

O presente trabalho aborda um estudo sobre a percepção do vozeamento das consoantes plosivas

(1) Fonoaudióloga; Clínica de Fonoaudiologia Maria Teresa Rosangela Lofredo - Bonatto, SP; Doutora em Lingüística Aplicada e Estudos da Linguagem pela Pontifícia Universidade Católica de São Paulo.

(2) Foneticista; Professora Titular do Departamento de Lingüística Aplicada da Faculdade de Comunicação e Filosofia da Pontifícia Universidade Católica de São Paulo, PUC-SP, São Paulo, SP; Doutora em Lingüística Aplicada e Estudos da Linguagem pela Pontifícia Universidade Católica de São Paulo.

Dados integrantes da pesquisa - Vozes Infantis: A Caracterização do Contraste de Vozeamento das Consoantes Plosivas no Português Brasileiro na Fala de Crianças de 3 a 12 anos [Tese de Doutorado]. Auxílio à Pesquisa: Capes Flexibilizada. na produção de fala de crianças de 3 anos. A motivação para a pesquisa surgiu do fato de terem sido encontradas, na inspeção espectrográfica da fala das crianças dessa faixa etária, características que ocorreram em algumas línguas do mundo ${ }^{1-3}$, mas não são comumente encontradas na fala do adulto 4,5 falante nativo do Português Brasileiro (PB). Tais características referem-se à presença da breathy vowel, entre a vogal e a liberação da oclusão, alterações na barra de sonoridade, presença de aspiração, como também produções de fricativas ou aproximantes em lugar das plosivas ${ }^{6}$.

Não existem muitos estudos na literatura fonética acerca da investigação de percepção do contraste de vozeamento. Com o objetivo de verificar como crianças, da faixa etária entre 5 e 7 anos percebiam as diferentes categorias de Voice Onset Time 
(doravante VOT) do inglês americano ${ }^{7}$, foi realizado um trabalho que constou de três experimentos. No primeiro as crianças deveriam realizar testes de identificação e discriminação das sílabas sintetizadas $/ \mathrm{ba} / \mathrm{e} / \mathrm{pa} / \mathrm{em}$ uma faixa de $10 \mathrm{~ms}$, variando entre $-10 \mathrm{~ms} \mathrm{e}+70 \mathrm{~ms}$. No segundo experimento, as crianças deveriam realizar as mesmas tarefas para o /da/ e /ta/ e no terceiro, grupo controle, apenas com crianças da faixa etária dos 7 anos, a tarefa de discriminar ocorreu antes de identificar, com o objetivo de controlar se a ordem delas poderia alterar os resultados. Concluiu-se que em ambos os experimentos a forma de percepção da criança era semelhante a dos adultos, com a fronteira entre 25 $\mathrm{ms}$ para / $/ \mathrm{p} / \mathrm{e} / \mathrm{b} / \mathrm{e}$ aproximadamente $40 \mathrm{~ms}$ para 0 /d/ e/t/ e que não havia diferença entre a percepção dos grupos etários e que a ordem das tarefas não interferia nos resultados.

Num outro estudo ${ }^{8}$, cinco crianças da faixa etária de três anos deveriam, associar as frases "This is a time" e "This is a dime", respectivamente, com um cartas de um relógio e outro de uma moeda. Foram gravados seis estímulos, para os quais o VOT variou entre $-30 \mathrm{~ms},-5 \mathrm{~ms},+20 \mathrm{~ms}+45 \mathrm{~ms}$, $+70 \mathrm{~ms} \mathrm{e}+100 \mathrm{~ms}$. Estes estímulos foram apresentados com intervalos de 11 segundos. Concluiu-se que, apesar da falta de motivação e atenção por parte das crianças, a discriminação de /t/ e /d/ era semelhante à encontrada para o adulto.

Em um trabalho ${ }^{4}$ sobre o vozeamento realizada por meio do VOT no PB, em falantes adultos, comparou-se a percepção dos estímulos de fala com a fala para o qual foi suprimida a porção positiva do VOT. Concluiu-se que a supressão do VOT positivo em monossílabos não-vozeados gerava erros de identificação pela troca de seu correspondente vozeado, mas alguns ainda, puderam ser identificados como não-vozeados, mesmo na presença do VOT zero.

Também em uma outra pesquisa ${ }^{9} \mathrm{com}$ adultos, em que foi realizada uma avaliação perceptivo-auditiva das plosivas de [pe], [be] e [da] com os VOTs sintetizados para o valor zero, foi constatado como resultado que as sílabas pesquisadas tinham sido percebidas pelos juízes como não-vozeadas.

Para este estudo partiu-se das seguintes hipóteses:

- A presença de aspiração não interfere na identificação da plosiva não-vozeada ${ }^{10}$.

- A presença da breathy vowel interfere na identificação da plosiva não-vozeada.

- Dependendo da porcentagem da interrupção da barra de sonoridade interferirá na percepção do vozeamento ${ }^{3,5,11-14}$.
O objetivo do presente estudo foi verificar a percepção auditiva que os adultos têm sobre as características das plosivas vozeadas e não-vozeadas produzidas por crianças da faixa etária de 3 anos.

\section{MÉTODOS}

Neste estudo foram selecionadas amostras de produção de fala de três repetições de frases-veículo (Diga baixinho.) com as palavraschave: "papa, baba, tata, dada, caca e gaga", que foram produzidas por quatro crianças da faixa etária de três anos, sendo três meninos e uma menina, de tal forma que se pudesse formar quatro grupos de estímulos, utilizando em cada um deles repetições diferentes de três crianças.

Optou-se por formar os grupos dessa forma para que se pudesse utilizar todas as emissões das crianças, mas sem contaminar as respostas dos juízes com estímulos excessivos. Quando alguma emissão apresentava titubeio ou precisão reduzida, procedia-se à substituição por outro da própria criança para evitar a interferência no julgamento dos juízes.

Cada uma das quatro listas de estímulos foi composta por 18 frases-veículo e por três frases distratoras com as palavras "gapa, taga, daca". Essas palavras foram "geradas" a partir das emissões das próprias crianças, no programa PRAAT (disponível no endereço eletrônico: www.praat.org), utilizandose procedimentos básicos para síntese de fala ${ }^{15}$.

Participaram como juízes do teste 144 sujeitos, estudantes universitários e professores de graduação e pós-graduação da Pontifícia Universidade Católica de São Paulo, dos quais, foram descartados 24 deles, por terem apresentado respostas inconsistentes ou manifestado dificuldades para a compreensão e realização do teste.

Cada juiz ouviu individualmente, com o auxílio de fones de ouvido, os 21 estímulos que estavam armazenados em um $C D$ e foram executados no programa Praat, com intervalo pré-determinado de cinco segundos ${ }^{16-21}$ e foram orientados que não seria possível interromper a sequência, mas que poderiam ouvir novamente, caso tivessem dúvidas com algum deles. Também foram alertados da presença dos logatomas isto é, que poderiam ouvir tanto frases-veículos com palavras do léxico como "jogo" como palavras com logatomas, isto é que possuem letras do PB, mas que não fazem parte do léxico como por exemplo "gojo". Os estímulos percebidos foram registrados em uma folha de resposta que identificava o juiz e a lista a que pertencia.

A contabilização das ocorrências foi realizada, primeiro para as sílabas em posição acentual tônica e depois para as pós-tônicas, eliminando-se as fra- 
ses com os distratores e considerando-se os julgamentos em vozeadas e não-vozeadas das demais frases, por exemplo, frase 2 plosiva /p/, frase 3 plosiva /d/ etc.

Os estímulos utilizados no teste fizeram parte do projeto que foi julgado pelo Comitê de Ética da PUC-SP sob o $n^{\circ}$ 19/2007, tendo sido considerado sem risco e com a necessidade de consentimento livre e esclarecido.

Após o levantamento das respostas, por frases, em cada grupo, foi realizada a contagem total por plosivas e, a partir daí, a contabilização total por meio de matrizes de confusão para todas as ocorrências. Esses dados foram submetidos à análise estatística, por meio dos testes ANOVA e teste t, com nível de significância $p<0,05$, para correlacionar as variáveis de vozeamento com a percepção dos juízes.

\section{RESULTADOS}

Os resultados do teste de percepção indicaram que houve um índice de acerto alto (de 92,5\% a
$97,5 \%)$ para a identificação das plosivas vozeadas em posição acentual tônica e, um nível médio de acerto para a identificação das plosivas não-vozeadas (de $65 \%$ a $67,7 \%$ ), ou seja, as produções das crianças da faixa etária de 3 anos favoreceram a percepção das plosivas como vozeadas (Tabelas 1 e 2).

Em relação às pós-tônicas, os resultados das respostas do teste de percepção de vozeamento, indicaram que houve um índice de acerto alto (de $92,2 \%$ a $94,4 \%$ ) para a identificação das plosivas vozeadas ${ }^{13,21-26}$ e um nível médio para a identificação das plosivas não-vozeadas de $66,7 \%$ a $76,7 \%$, como indicado nas Tabelas 3 e 4.

Esses resultados foram submetidos a análise estatística que correlacionou a duração da barra de sonoridade (SON) e a duração do silêncio ((SIL) com o número de respostas que identificaram o estímulo como vozeado (PVOZ), como não-vozeado (PÑVOZ) e a porcentagem de sonoridade para cada plosiva (PSON) e que podem ser vistos nas Tabelas de 5 a 8 .

Tabela 1 - Contabilização geral e porcentagem do número de ocorrências das plosivas quanto à percepção do vozeamento em posição acentual tônica

\begin{tabular}{cccccc}
\hline Plosiva & não-vozeada & $\%$ & vozeada & $\%$ & total \\
\hline $\mathrm{p}$ & 234 & 65 & 126 & 35 & 360 \\
$\mathrm{t}$ & 264 & 73,3 & 96 & 26,7 & 360 \\
$\mathrm{k}$ & 276 & 76,7 & 84 & 23,3 & 360 \\
$\mathrm{~b}$ & 17 & 4,7 & 343 & 95,3 & 360 \\
$\mathrm{~d}$ & 27 & 7,5 & 333 & 92,5 & 360 \\
$\mathrm{~g}$ & 9 & 2,5 & 351 & 97,5 & 360 \\
\hline
\end{tabular}

Tabela 2 - Matriz de confusão que apresenta os resultados do teste para a percepção do vozeamento para as plosivas em posição acentual tônica

\begin{tabular}{ccccccc}
\hline $\mathbf{R} / \mathbf{E} \rightarrow$ & $\mathbf{p}$ & $\mathbf{t}$ & $\mathbf{k}$ & $\mathbf{b}$ & $\mathbf{d}$ & $\mathbf{g}$ \\
\hline $\mathbf{p}$ & $\mathbf{2 3 2}$ & 21 & 1 & 17 & 1 & 1 \\
$\mathbf{t}$ & 1 & $\mathbf{2 3 6}$ & 5 & 0 & 21 & 0 \\
$\mathbf{k}$ & 1 & 7 & $\mathbf{2 6 8}$ & 0 & 5 & 8 \\
$\mathbf{b}$ & 106 & 1 & 1 & $\mathbf{3 3 0}$ & 17 & 5 \\
$\mathbf{d}$ & 2 & 90 & 3 & 2 & $\mathbf{2 9 8}$ & 30 \\
$\mathbf{g}$ & 14 & 5 & 79 & 9 & 18 & $\mathbf{2 9 6}$ \\
$\mathbf{p l}$ & 0 & 0 & 0 & 1 & 0 & 0 \\
$\mathbf{k l}$ & 0 & 0 & 1 & 0 & 0 & 0 \\
$\mathbf{g l}$ & 0 & 0 & 1 & 0 & 0 & 0 \\
$\mathbf{v}$ & 4 & 0 & 0 & 1 & 0 & 20 \\
$\mathbf{m}$ & 0 & 0 & 0 & 1 & 0 & 0 \\
\hline total= & $\mathbf{3 6 0}$ & $\mathbf{3 6 0}$ & $\mathbf{3 6 0}$ & $\mathbf{3 6 0}$ & $\mathbf{3 6 0}$ & $\mathbf{3 6 0}$ \\
\hline
\end{tabular}


Tabela 3 - Contabilização geral e porcentagem do número de ocorrências das plosivas quanto à percepção do vozeamento em posição acentual pós-tônica

\begin{tabular}{cccccc}
\hline Plosiva & não-vozeada & $\%$ & vozeada & $\%$ & total \\
\hline $\mathrm{p}$ & 240 & 66,7 & 120 & 33,3 & 360 \\
$\mathrm{t}$ & 276 & 76,7 & 84 & 23,3 & 360 \\
$\mathrm{k}$ & 255 & 70,8 & 105 & 29,2 & 360 \\
$\mathrm{~b}$ & 20 & 5,6 & 340 & 94,4 & 360 \\
$\mathrm{~d}$ & 28 & 7,5 & 332 & 92,2 & 360 \\
$\mathrm{~g}$ & 21 & 5,8 & 339 & 94,2 & 360 \\
\hline
\end{tabular}

Tabela 4 - Matriz de confusão que apresenta os resultados do teste de percepção do vozeamento para as plosivas em posição acentual pós-tônica

\begin{tabular}{ccccccc}
\hline $\mathbf{R} / \mathbf{E} \rightarrow$ & $\mathbf{p}$ & $\mathbf{t}$ & $\mathbf{k}$ & $\mathbf{b}$ & $\mathbf{d}$ & $\mathbf{g}$ \\
\hline $\mathbf{p}$ & $\mathbf{2 3 1}$ & 14 & 1 & 19 & 0 & 1 \\
$\mathbf{t}$ & 6 & $\mathbf{2 5 5}$ & 9 & 1 & 26 & 1 \\
$\mathbf{k}$ & 3 & 7 & $\mathbf{2 4 3}$ & 0 & 2 & 19 \\
$\mathbf{b}$ & 99 & 1 & 4 & $\mathbf{3 1 1}$ & 15 & 5 \\
$\mathbf{d}$ & 2 & 78 & 3 & 5 & $\mathbf{2 9 1}$ & 29 \\
$\mathbf{g}$ & 16 & 5 & 96 & 9 & 22 & $\mathbf{3 0 4}$ \\
$\mathbf{v}$ & 3 & 0 & 0 & 3 & 2 & 1 \\
$\mathbf{z}$ & 0 & 0 & 2 & 0 & 0 & 0 \\
$\mathbf{c h}$ & 0 & 0 & 2 & 0 & 0 & 0 \\
$\mathbf{j}$ & 0 & 0 & 0 & 3 & 0 & 0 \\
$\mathbf{I}$ & 0 & 0 & 0 & 4 & 0 & 0 \\
$\mathbf{m}$ & 0 & 0 & 0 & 2 & 0 & 0 \\
$\mathbf{r}$ & 0 & 0 & 0 & 1 & 1 & 0 \\
$\mathbf{y a}$ & 0 & 0 & 0 & 1 & 0 & 0 \\
wa & 0 & 0 & 0 & 1 & 0 & 0 \\
$\mathbf{g l}$ & 0 & 0 & 0 & 0 & 1 & 0 \\
total= & $\mathbf{3 6 0}$ & $\mathbf{3 6 0}$ & $\mathbf{3 6 0}$ & $\mathbf{3 6 0}$ & $\mathbf{3 6 0}$ & $\mathbf{3 6 0}$ \\
\hline
\end{tabular}

Sabe-se pela literatura fonética ${ }^{27}$, que as vogais que antecedem os sons vozeados apresentam durações mais longas do que as antecedem os sons não-vozeados. Com base nesses conhecimentos, foi realizada, por meio do teste t, com nível de significância de $p<0,05$ outra análise estatística, com $o$ objetivo de verificar se na produção das plosivas da faixa etária de 3 anos, essa diferenciação também se encontrava presente.

Os resultados da aplicação do teste t revelaram para a faixa etária de 3 anos, diferenças significativas apenas entre a duração do /a/ tônico antes do $/ \mathrm{p} /$ e do /b/, mas não entre a duração do /a/ tônico antes do /t/ e /d/ e antes de / $/ \mathrm{e} / \mathrm{g}$. 
Tabela 5 - Porcentagem de sonoridade correlacionada à percepção de vozeamento - posição acentual tônica - plosivas vozeadas

\begin{tabular}{|c|c|c|c|c|c|c|c|}
\hline PLO & CRI & REP & SON & SIL & PVOZ & P ñ voz & PSON \% \\
\hline \multirow[t]{12}{*}{$/ b /$} & 1 & 1 & 65 & 36 & 28 & 2 & 64,4 \\
\hline & & 2 & 70 & 27 & 28 & 2 & 72,2 \\
\hline & & 3 & 52 & 83 & 20 & 10 & 38,5 \\
\hline & 2 & 1 & 86 & 0 & 30 & 0 & 100 \\
\hline & & 2 & 132 & 0 & 30 & 0 & 100 \\
\hline & & 3 & 134 & 0 & 30 & 0 & 100 \\
\hline & 3 & 1 & 50 & 0 & 30 & 0 & 100 \\
\hline & & 2 & 60 & 54 & 30 & 0 & 52,6 \\
\hline & & 3 & 84 & 22 & 30 & 0 & 79,3 \\
\hline & 4 & 1 & 86 & 0 & 27 & 3 & 100 \\
\hline & & 2 & 96 & 0 & 30 & 0 & 100 \\
\hline & & 3 & 95 & 0 & 28 & 0 & 100 \\
\hline \multirow[t]{12}{*}{$/ d /$} & 1 & 1 & 55 & 63 & 29 & 1 & 46,6 \\
\hline & & 2 & 45 & 46 & 29 & 1 & 49,5 \\
\hline & & 3 & 56 & 82 & 27 & 3 & 40,6 \\
\hline & 2 & 1 & 74 & 0 & 30 & 0 & 100 \\
\hline & & 2 & 94 & 0 & 30 & 0 & 100 \\
\hline & & 3 & 94 & 0 & 30 & 0 & 100 \\
\hline & 3 & 1 & 59 & 36 & 30 & 0 & 62,1 \\
\hline & & 2 & 36 & 72 & 24 & 6 & 33,3 \\
\hline & & 3 & 64 & 29 & 30 & 0 & 68,8 \\
\hline & 4 & 1 & 87 & 0 & 28 & 2 & 100 \\
\hline & & 2 & 74 & 0 & 30 & 0 & 100 \\
\hline & & 3 & 123 & 0 & 28 & 2 & 100 \\
\hline \multirow[t]{12}{*}{$/ g /$} & 1 & 1 & 31 & 59 & 27 & 3 & 34,4 \\
\hline & & 2 & 44 & 43 & 30 & 0 & 50,6 \\
\hline & & 3 & 43 & 20 & 30 & 0 & 68,3 \\
\hline & 2 & 1 & 93 & 26 & 30 & 0 & 78,2 \\
\hline & & 2 & 81 & 0 & 30 & 0 & 100 \\
\hline & & 3 & 106 & 30 & 30 & 0 & 77,9 \\
\hline & 3 & 1 & 75 & 35 & 29 & 1 & 68,2 \\
\hline & & 2 & 75 & 35 & 30 & 0 & 68,2 \\
\hline & & 3 & 75 & 35 & 30 & 0 & 68,2 \\
\hline & 4 & 1 & 102 & 0 & 30 & 0 & 100 \\
\hline & & 2 & 78 & 30 & 30 & 0 & 72,2 \\
\hline & & 3 & 76 & 0 & 27 & 3 & 100 \\
\hline
\end{tabular}

Legenda: PLO - plosiva; CRI - criança; REP - repetição; SON - duração da barra de sonoridade; SIL. - duração do silêncio; PVOZ - julgamento perceptivo da plosiva como vozeada; PÑVOZ - julgamento perceptivo da plosiva como não-vozeada; PSON - porcentagem de sonoridade 
Tabela 6 - Porcentagem de sonoridade correlacionada à percepção de vozeamento - posição acentual tônica - plosivas não-vozeadas

\begin{tabular}{|c|c|c|c|c|c|c|c|}
\hline PLO & CRI & REP & SON & SIL & PVOZ & p ñ voz & PSON \% \\
\hline \multirow[t]{12}{*}{$/ \mathrm{p} /$} & 1 & 1 & 35 & 20 & 29 & 1 & 63,6 \\
\hline & & 2 & 64 & 60 & 18 & 12 & 51,6 \\
\hline & & 3 & 55 & 0 & 28 & 2 & 100 \\
\hline & 2 & 1 & 25 & 124 & 5 & 25 & 16,8 \\
\hline & & 2 & 13 & 106 & 10 & 20 & 10,9 \\
\hline & & 3 & 28 & 48 & 12 & 18 & 36,8 \\
\hline & 3 & 1 & 36 & 71 & 5 & 25 & 33,6 \\
\hline & & 2 & 30 & 113 & 6 & 24 & 21 \\
\hline & & 3 & 32 & 57 & 5 & 25 & 36 \\
\hline & 4 & 1 & 45 & 59 & 4 & 26 & 43,3 \\
\hline & & 2 & 31 & 41 & 4 & 26 & 43,1 \\
\hline & & 3 & 25 & 99 & 0 & 30 & 20,2 \\
\hline \multirow[t]{12}{*}{$/ t /$} & 1 & 1 & 47 & 13 & 20 & 10 & 78 \\
\hline & & 2 & 54 & 71 & 19 & 11 & 43,2 \\
\hline & & 3 & 54 & 71 & 25 & 5 & 43,2 \\
\hline & 2 & 1 & 17 & 133 & 1 & 29 & 11,3 \\
\hline & & 2 & 18 & 89 & 4 & 26 & 16,8 \\
\hline & & 3 & 13 & 83 & 4 & 26 & 13,5 \\
\hline & 3 & 1 & 19 & 82 & 2 & 28 & 18,8 \\
\hline & & 2 & 24 & 87 & 17 & 13 & 21,6 \\
\hline & & 3 & 17 & 80 & 1 & 29 & 17,5 \\
\hline & 4 & 1 & 23 & 128 & 2 & 28 & 15,2 \\
\hline & & 2 & 43 & 140 & 0 & 30 & 23,5 \\
\hline & & 3 & 40 & 120 & 1 & 29 & 25 \\
\hline \multirow[t]{12}{*}{$/ \mathrm{k} /$} & 1 & 1 & 24 & 69 & 24 & 6 & 29 \\
\hline & & 2 & 37 & 54 & 24 & 6 & 40,7 \\
\hline & & 3 & 36 & 73 & 16 & 14 & 33 \\
\hline & 2 & 1 & 0 & 138 & 1 & 29 & 0 \\
\hline & & 2 & 0 & 99 & 8 & 22 & 0 \\
\hline & & 3 & 0 & 140 & 1 & 29 & 0 \\
\hline & 3 & 1 & 0 & 136 & 9 & 30 & 0 \\
\hline & & 2 & 23 & 116 & 4 & 26 & 16,6 \\
\hline & & 3 & 0 & 149 & 0 & 30 & 0 \\
\hline & 4 & 1 & 32 & 163 & 4 & 26 & 16,4 \\
\hline & & 2 & 0 & 140 & 0 & 30 & 0 \\
\hline & & 3 & 19 & 109 & 2 & 28 & 14,8 \\
\hline
\end{tabular}

Legenda: PLO - plosiva; CRI - criança; REP - repetição; BV (ms) breathy vowel e barra de sonoridade; SIL. - duração do silêncio; PVOZ - julgamento perceptivo da plosiva como vozeada; PÑVOZ - julgamento perceptivo da plosiva como não-vozeada; PSON - porcentagem de sonoridade 
Tabela 7 - Porcentagem de sonoridade correlacionada à percepção de vozeamento - posição acentual pós-tônica - plosivas vozeadas

\begin{tabular}{|c|c|c|c|c|c|c|c|}
\hline PLO & CRI & REP & SON & SIL & PVOZ & PNVOZ & PSON \% \\
\hline \multirow[t]{12}{*}{$/ \mathbf{b} /$} & 1 & 1 & 56 & 29 & 27 & 3 & 65,9 \\
\hline & & 2 & 49 & 51 & 27 & 3 & 49 \\
\hline & & 3 & 52 & 70 & 20 & 10 & 42,6 \\
\hline & 2 & 1 & 72 & 0 & 30 & 0 & 100 \\
\hline & & 2 & 123 & 0 & 29 & 1 & 100 \\
\hline & & 3 & 80 & 0 & 30 & 0 & 100 \\
\hline & 3 & 1 & 60 & 0 & 27 & 3 & 100 \\
\hline & & 2 & 71 & 0 & 30 & 0 & 100 \\
\hline & & 3 & 69 & 41 & 30 & 0 & 62,7 \\
\hline & 4 & 1 & 98 & 0 & 30 & 0 & 100 \\
\hline & & 2 & 73 & 0 & 27 & 3 & 100 \\
\hline & & 3 & 90 & 0 & 30 & 0 & 100 \\
\hline \multirow[t]{12}{*}{$/ d /$} & 1 & 1 & 45 & 45 & 29 & 1 & 50 \\
\hline & & 2 & 32 & 40 & 28 & 2 & 44,4 \\
\hline & & 3 & 37 & 73 & 26 & 4 & 33,6 \\
\hline & 2 & 1 & 85 & 0 & 30 & 0 & 100 \\
\hline & & 2 & 75 & 0 & 30 & 0 & 100 \\
\hline & & 3 & 75 & 0 & 30 & 0 & 100 \\
\hline & 3 & 1 & 70 & 0 & 29 & 1 & 100 \\
\hline & & 2 & 55 & 0 & 28 & 2 & 100 \\
\hline & & 3 & 65 & 27 & 27 & 3 & 70,7 \\
\hline & 4 & 1 & 75 & 0 & 29 & 1 & 100 \\
\hline & & 2 & 84 & 0 & 29 & 1 & 100 \\
\hline & & 3 & 138 & 0 & 29 & 1 & 100 \\
\hline \multirow[t]{12}{*}{$/ g /$} & 1 & 1 & 28 & 57 & 28 & 2 & 32,9 \\
\hline & & 2 & 42 & 51 & 16 & 14 & 52,9 \\
\hline & & 3 & 29 & 75 & 26 & 4 & 27,9 \\
\hline & 2 & 1 & 81 & 0 & 30 & 0 & 100 \\
\hline & & 2 & 32 & 108 & 30 & 0 & 22,9 \\
\hline & & 3 & 91 & 0 & 30 & 0 & 100 \\
\hline & 3 & 1 & 61 & 24 & 25 & 5 & 71,8 \\
\hline & & 2 & 26 & 47 & 28 & 2 & 71,8 \\
\hline & & 3 & 61 & 24 & 29 & 1 & 71,8 \\
\hline & 4 & 1 & 75 & 0 & 30 & 0 & 100 \\
\hline & & 2 & 132 & 0 & 30 & 0 & 100 \\
\hline & & 3 & 45 & 45 & 26 & 4 & 50 \\
\hline
\end{tabular}

Legenda: PLO - plosiva; CRI - criança; REP - repetição; SON - duração da barra de sonoridade; SIL.- duração do silêncio; PVOZ - julgamento perceptivo da plosiva como vozeada; PÑVOZ - julgamento perceptivo da plosiva como não-vozeada; PSON \% porcentagem de sonoridade 
Tabela 8 - Porcentagem de sonoridade correlacionada à percepção do vozeamento - posição acentual pós-tônica - plosivas não-vozeadas

\begin{tabular}{|c|c|c|c|c|c|c|c|}
\hline PLO & CRI & REP & SON & SIL & PVOZ & PNVOZ & PSON \\
\hline \multirow[t]{12}{*}{$/ p /$} & 1 & 1 & 38 & 20 & 28 & 2 & 65,5 \\
\hline & & 2 & 56 & 43 & 17 & 13 & 56,6 \\
\hline & & 3 & 52 & 0 & 26 & 4 & 100 \\
\hline & 2 & 1 & 17 & 108 & 6 & 24 & 13,6 \\
\hline & & 2 & 23 & 101 & 8 & 22 & 18,6 \\
\hline & & 3 & 9 & 109 & 11 & 19 & 7,6 \\
\hline & 3 & 1 & 25 & 88 & 4 & 26 & 22,1 \\
\hline & & 2 & 20 & 75 & 5 & 25 & 21,1 \\
\hline & & 3 & 20 & 96 & 7 & 23 & 17,2 \\
\hline & 4 & 1 & 17 & 78 & 5 & 25 & 17,9 \\
\hline & & 2 & 33 & 75 & 3 & 27 & 30,6 \\
\hline & & 3 & 71 & 42 & 30 & 0 & 62,8 \\
\hline \multirow[t]{12}{*}{$/ t /$} & 1 & 1 & 58 & 51 & 19 & 11 & 53,2 \\
\hline & & 2 & 35 & 88 & 18 & 12 & 28,5 \\
\hline & & 3 & 35 & 88 & 18 & 12 & 28,5 \\
\hline & 2 & 1 & 15 & 109 & 1 & 29 & 12,1 \\
\hline & & 2 & 0 & 101 & 3 & 27 & 0 \\
\hline & & 3 & 0 & 147 & 1 & 29 & 0 \\
\hline & 3 & 1 & 15 & 77 & 3 & 27 & 16,3 \\
\hline & & 2 & 26 & 101 & 17 & 13 & 20,5 \\
\hline & & 3 & 17 & 80 & 0 & 30 & 17,5 \\
\hline & 4 & 1 & 24 & 134 & 1 & 29 & 15,2 \\
\hline & & 2 & 36 & 47 & 3 & 27 & 43,4 \\
\hline & & 3 & 41 & 83 & 0 & 30 & 33 \\
\hline \multirow[t]{12}{*}{$/ \mathrm{k} /$} & 1 & 1 & 29 & 76 & 22 & 8 & 27,6 \\
\hline & & 2 & 36 & 41 & 22 & 8 & 46,7 \\
\hline & & 3 & 40 & 32 & 17 & 13 & 55,5 \\
\hline & 2 & 1 & 52 & 49 & 25 & 5 & 51,5 \\
\hline & & 2 & 0 & 126 & 8 & 22 & 0 \\
\hline & & 3 & 0 & 126 & 1 & 29 & 0 \\
\hline & 3 & 1 & 14 & 99 & 2 & 28 & 12,4 \\
\hline & & 2 & 27 & 63 & 1 & 29 & 30 \\
\hline & & 3 & 19 & 74 & 0 & 30 & 20,4 \\
\hline & 4 & 1 & 25 & 90 & 6 & 24 & 21,7 \\
\hline & & 2 & 39 & 106 & 1 & 29 & 26,9 \\
\hline & & 3 & 27 & 107 & 0 & 30 & 20,2 \\
\hline
\end{tabular}

Legenda: PLO - plosiva; CRI - criança; REP - repetição; BV (ms) duração da breathy vowel e barra de sonoridade; SIL. - duração do silêncio; PVOZ - julgamento perceptivo da plosiva como vozeada; PÑVOZ - julgamento perceptivo da plosiva como não-vozeada; PSON \% porcentagem de sonoridade. 


\section{DISCUSSÃO}

Nesta pesquisa, julgou-se que nessa faixa etária essa tendência se deva a uma determinada latência que caracteriza a produção dos gestos articulatórios e glotais. Desse modo, a interrupção do gesto glotal é retardada, o que se manifesta na produção de vogais caracterizadas por uma breathy vowel na porção final da vogal ou pela manutenção da vibração das pregas vocais em parte do intervalo de obstrução entre articuladores, conforme foi possível inferir a partir da inspeção dos espectrogramas de banda larga. A presença de aspiração pode ser compreendida de forma análoga a retomada do vozeamento, após a interrupção também se apresentou em muitos casos retardada, como se pode verificar em algumas produções das consoantes plosivas nessa faixa etária.

Os resultados dos testes de percepção evidenciaram que, diferentemente de algumas pesquisas ${ }^{3,22,26,28}$ não houve necessidade de serem preenchidos pelo menos $80 \%$ do intervalo de obstrução com vozeamento.

As plosivas vozeadas em posição acentual tônica apresentaram predominantemente porcentagem de pré-vozeamento superior a $53 \%$. Foram verificados também alguns valores de porcentagem entre $33,3 \%$ e $52,6 \%$, mas mesmo nesses casos, em que essa porcentagem variou em valores abaixo de $53 \%$, houve predominância de respostas apontando a percepção de sons vozeados.

As plosivas não-vozeadas em posição acentual tônica que apresentaram porcentagem abaixo de $30 \%$ de pré-vozeamento foram identificadas predominantemente como não-vozeadas. Quando os valores de porcentagem estiveram entre $30 \%$ e $50 \%$ ora favoreceram julgamentos de vozeamento ora de não-vozeamento. Acima de $51,6 \%$ foram identificadas como vozeadas. Apenas uma repetição com $29 \%$ foi identificada como vozeada.

As plosivas vozeadas em posição acentual pós-tônica apresentaram resultados que apontaram a mesma direção, isto é, a predominância da porcentagem de pré-vozeamento superior a $53 \%$. Encontrou-se valores abaixo dessa porcentagem, que variaram entre $22,9 \%$ e $52,9 \%$ para os quais houve predominância de respostas apontando a percepção de sons vozeados.

As plosivas não-vozeadas, nessa posição acentual, que apresentaram porcentagem abaixo de $30 \%$, também foram identificadas predominantemente como não-vozeadas e as com valores que variaram entre $30 \%$ e $50 \%$, ora favoreceram os julgamentos de vozeamento, ora de não-vozeamento. Acima de 51,5\%, houve predominância de julgamentos como vozeadas.

\section{CONCLUSÃO}

Os resultados deste estudo evidenciam a relevância da análise fonético-acústica para a compreensão das relações entre produção e percepção da fala, visto que permitiu evidenciar estratégias de produção das crianças da faixa etária de 3 anos e correlacioná-las aos efeitos que tiveram na percepção de adultos falantes nativos do PB.

Em relação às consoantes plosivas não-vozeadas com presença produtiva da aspiração, que não tem papel distintivo no PB, este estudo sugere que ocorreram dificuldades na sincronização entre o gesto glotal e o articulatório, mas que não interferiram na percepção desses sons, uma vez que as plosivas não-vozeadas que apresentaram aspiração foram corretamente avaliadas como não-vozeadas. 


\begin{abstract}
Purpose: to submit a study on the auditory perception that adults have on the characteristics of voiced and unvoiced stops produced by 3-year-old children. Methods: first, the characteristics found in the production of stops were acoustically inspected in relation to (1) obstruction interval, (2) burst, and (3) quantitative analysis of Voice Onset Time (VOT), measured in six plosives found in Brazilian Portuguese (BP). The carrier sentence "Diga___ baixinho", produced by four 3-year-old children - three male and one female, Brazilian Portuguese native speakers - was submitted to wide-band spectrographic analysis using PRAAT software. Second, the corpus produced by the children was submitted to 120 adult individuals in an identification type perception test. Results: the production showed uncommon characteristics for adult speech in relation to both voiced and unvoiced stops. The test showed that the adults perceived the unvoiced stops as voiced due to the presence of breathy vowel. The voiced plosives were always correctly identified. The articulatory position of the bilabial, alveolar and velar stops did not interfere in the way the stops were judged. The ANOVA test (sig $=0,05)$ showed that the plosives were correctly identified when they presented breathy vowel or voicing with over $60 \%$ of voicing. The presence of aspiration did not interfere in the unvoiced stops judgment. Conclusion: the results indicate the relevance of acoustic phonetics analysis in studies on the production and the perception of speech, which may help to elaborate therapeutical procedures and facilitate the follow-up of their development.
\end{abstract}

KEYWORDS: Child; Speech; Sound Spectrography; Speech Perception

\section{REFERÊNCIAS}

1. Lisker L, Abramson A. A cross-language study of voicing in initial stops: acoustical measurements. Word J Linguistic Circle. 1964; 20(3):384-422.

2. Ladefoged P, Maddieson I. The sound's of the world's languages. Massachusetts: Blackwell Publishers Inc; 1996.

3. Dommelen WA. Preaspiration in intervocalic $|k| x$ Ig| in nowergian. ICPhs. 1999; 3:2037-40.

4. Behlau MS. Análise do tempo de início da sonorização na discriminação dos sons plosivos do Português. [tese] São Paulo (SP): Universidade Federal de São Paulo; 1986.

5. Amino K, Ji SR, Shinohara SH. Perception of Japanese plosives by Korean speakers. XVI International Congress of Phonetic Science - ICPhsSaarbrticken; 2007: 1788-92.

6. Bonatto MTRL. Vozes infantis: a caracterização do contraste de vozeamento das consoantes no Português brasileiro na fala de crianças de 3 a 12 anos. [tese] São Paulo (SP): Pontifícia Universidade Católica; 2007.

7. Wolf CG. The perception of stop consonants by children. J Experim Child Psychol. 1973; 16(2):318-31.

8. Weinterhorn JMS, MacNeilage RF, Preston MS. Perception of voiced and voiceless stops consonants in three year old children. Status Report on Speech Research. 1976; 41-5.
9. Camargo ZA. Análise da qualidade vocal de um grupo de indivíduos disfônicos: uma abordagem interpretativa e integrada de dados de natureza acústica, perceptiva e eletroglotográfica. [tese]. São Paulo (SP): Pontifícia Universidade Católica; 2002. 10. Zitta SM. Análise perceptivo-auditiva e acústica em mulheres com nódulos vocais [dissertação] Curitiba (PR): Centro Federal de Educação Tecnológica do Paraná; 2005.

11. Ficker LB. Produção e percepção das plosivas do Português brasileiro: estudo fonético-acústico da fala de um sujeito com deficiência auditiva. [tese]. São Paulo (SP): Pontifícia Universidade Católica; 2003.

12. Jessen M. An acoustic study of contrasting plosives and click accompaniments in Xhosa. Phonetica. 2002; 59(2-3):150-79.

13. Kuijpers CTL. Perception of the voicing contrast by Dutch children and adults. J Phonetics. 1996; 24(3):367-82.

14. Amino K, Sugawara T, Arai T. Idiosyncrasy of nasal sounds in human speaker identification and their acoustic properties. Acoust Scie Technol. 2006; 27(4):233-5.

15. Llisterri J. Técnicas sobre sínteses de manipulação de sinal sonoro para o desenvolvimento de provas de percepção de fala. [Curso] Tópicos em estudo sobre fala. Pontifícia Universidade Católica de São Paulo; 2005.

16. Veloso J. The role of consonantal duration and tenseness in the perception of voicing distinctions 
of portuguese stops. XIII International Congress of Phonetic Science Stockholm Proceeding Stockholm ICPhs; 1995. p.266-9.

17. Susskind JE. Children's perception of genderbased illusory correlations: enhancing preexisting relationships between gender and behavior. Sex Roles. 2003; 48(11-12): 483-94.

18. Michel U, Schmidt E, Batzler U. Results of psychological testing of patients aged 3-6 years. Eur J Pediatr. 1990; 149(Suppl1):34-8.

19. Keilmann A, Wintermeyer M. Is a specialised training of phonological awareness indicated in every preschool child? Folia Phoniatr Logop. 2008; 60(2):73-9.

20. Kingma $\mathrm{H}$. Thresholds for perception of direction of linear acceleration as a possible evaluation of the otolith function. BMC Ear Nose Throat Disord. 2005; 5(1):5.

21. Bloomfield FH, Shuan D, Perry D, Aftimos S. Isolated absence of the Moro reflex in a baby with Charge syndrome could reflect vestibular abnormalities. J Child Neurol. 2008; 23(5):561-3.

22. Gerrits E. Speech perception of young children at risk for dyslexia. XV International Congress of Phonetic Science Stockholm Proceeding Stockholm ICPhs; 1999.
23. Bucci MP, Kapoula Z, Yang Q, Bremond-Gignac $D$, Wiener-Vacher S. Speed-accuracy of saccades, vergence and combined eye movements in children with vertigo. Exp Brain Res. 2004; 157(3):286-95.

24. Medina V, Serniclaes W. Late development of the categorical perception of speech sounds in pre-adolescent children. ZAS Papers in Linguistics. 2005; 42:13-32.

25. Manis $F$, Keating $P$. Speech perception in dyslexic children with and without language impairments. UCLA. Working Papers In Phonetics. 2005; 103(3):30-47.

26. Pearce M. Language-specific contribution of Fo and VOT in the perception of voicing: L1, L2 and orthography. UCLWPL. 2005. Disponível em: URL: http://www.chc.ucl.ac.uk/events/files/CHC\%20post er\%20final\%20dark\%20a.pdf

27. Mendes BCA. Estudo fonético acústico das vogais do Português brasileiro: dados da produção e percepção da fala de um sujeito deficiente auditivo. [tese]. São Paulo (SP): Pontifícia Universidade Católica; 2003.

28. Kuzla C, Ernestus M. Prosodic conditioning of phonetic detail of German plosives. XVI International Congress of Phonetic Science - ICPhs. Saarbrticken; 2007.
RECEBIDO EM: 08/01/2008

ACEITO EM: 08/09/2008

Endereço para Correspondência:

Maria Teresa Rosangela Lofredo Bonatto

Av Paulista, 509 conj. 410

São Paulo - SP

CEP: 01311-910

E-mail: mteresabonatto@uol.com.br 